\title{
EVALUATION OF RETENTION OF TWO IMPLANT SUPPORTED CERAMIC CROWNS USING TWO TEMPORARY CEMENTS
}

\author{
Mohamed Tarek Farahat * and Ahmad Khaled Aboelfadl**
}

\begin{abstract}
Aim: The purpose of this in vitro study was to evaluate the effect of two temporary cements (Resin based cement and Eugenol Free Zinc oxide cement) on the retention of two titanium implant supported crowns made of (Polymer infiltrated hybrid glass ceramic and Yttrium stabilized tetragonal zirconia).

Materials and methods: 20 dummy implants were inserted in epoxy resin blocks. The twenty blocks were divided according to the crown material into two groups $(n=10)$ : Group I: VITA EN-

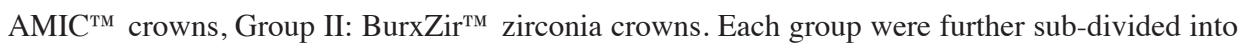
two sub-groups ( $\mathrm{n}=5)$, according to the temporary cement material used (Sub-group A: crowns cemented with DentoTemp ${ }^{\mathrm{TM}}$ cement, Sub group B: crowns cemented with Tempbond NE ${ }^{\mathrm{TM}}$ cement). The crowns were fabricated with CAD-CAM technology. Each crown had two wings on the mesial and distal surfaces to aid in retention analysis. The crowns were cemented to the titanium abutments and then retention was measured using uni-axial tensile force test.
\end{abstract}

Results: DentoTemp ${ }^{\mathrm{TM}}$ cement showed a higher retentive values with both crown materials. The retentive values of the DentoTemp ${ }^{\mathrm{TM}}$ temporary cement was significantly higher than those of the Tempbond NE ${ }^{\mathrm{TM}}$ cement when used with BruxZir ${ }^{\mathrm{TM}}$ Zirconia crowns.

Conclusions: Resin based temporary cement showed significantly higher retention results than non-eugenol temporary cement while the difference in crown material showed no significant difference.

KEYWORDS: Implants, Retention, Temporary cement, Zirconia, Hybrid ceramics.

\section{INTRODUCTION}

Dental implants are an effective and popular option for replacing the single missing tooth and form an important part of mainstream dental practice today. Their use often represents a better alternative over traditional options of tooth replacement. The selection of the method of crown retention presents the clinician with a treatment planning

* General Practitioner in Cairo Health Directorate, Ministry of Health

** Lecturer of Fixed Prosthodontics, Crown and Bridge Department Faculty of Dentistry, Ain Shams University. 
challenge that involves recognition of the drivers of the desired treatment outcome. Among other factors, aspects of retrievability versus aesthetics have largely been considered in deciding whether crowns should be screw-retained or cement-retained. ${ }^{1}$

Zirconia is one of the most commonly used all ceramic materials due to its high strength, fracture toughness, biocompatibility, and excellent esthetics, especially with the introduction of the new translucent CAD-CAM zirconia blanks, and with the introduction of the new polymer infiltrated hybrid glass ceramics as CAD-CAM blocks for fabricating implant supported restorations, many studies have been done to test its properties in vivo and in vitro because of their excellent esthetics, sufficient strength, high resilience and good shock absorbing capacity.

The option to cement crowns to implant abutments may be elected, or contrastingly forced upon the clinician due to implant positioning. The choice of cement must subsequently be considered. The majority of cements used in implant dentistry at present have been designed for use with crowns luted to natural teeth. ${ }^{1}$

In implant dentistry, careful consideration of the choice of cement should include reference to the abutment and crown specifications, opposing surface characteristics, desired retention and individual properties of the preferred cement. Different types of cements provide different levels of crown retention. ${ }^{2}$

The degree of retrievability of the implantsupported cement-retained prosthesis is inversely proportional to the retention strength of the cement used. At the same time, regardless of the cement type, provisional/temporary or permanent, and of the number and characteristics of the abutments, several mechanical and biological factors may affect the retentiveness of the cement in a given restoration. ${ }^{3}$
Urethane-based resin cement (temporary cement), resin-modified glass ionomer and resin composite cements (permanent cements) are the examples of available luting agents that are used clinically to cement crowns to implant abutments. ${ }^{3}$

The cement used for a cement-retained implant needs to provide sufficient retention of the superstructure to the abutment and also to allow for retrieval of the superstructure from the abutment if necessary. In order to satisfy these requirements, temporary cements can be favorably used for this type of implant prosthesis. ${ }^{4}$

The aim of this in vitro study was to evaluate the effect of two temporary crown cements (Resin based cement and Eugenol Free Zinc oxide cement) on the retention of two titanium implant supported crowns made of (Polymer infiltrated hybrid glass ceramic and Yttrium stabilized tetragonal zirconia).

\section{MATERIALS AND METHODS}

In this in-vitro study, 20 samples including 10 zirconia crowns and 10 Polymer infiltrated hybrid glass ceramic crowns were fabricated by $\mathrm{CAD} /$ CAM milling system cemented on titanium implant abutments. Two temporary cement types were used, resin based temporary cement and zinc oxide non eugenol temporary cement. the twenty all ceramic crowns were divided according to crown materials: Group $1(\mathrm{n}=10)$ : Polymer infiltrated hybrid glass ceramic (VITA ENAMIC ${ }^{\mathrm{TM}}$ ) crowns Group 2 $(\mathrm{n}=10)$ : Yttrium stabilized tetragonal zirconia (BruxZir $^{\mathrm{TM}}$ Solid zirconia) crowns. Each group was subdivided according to the cement type used into: Subgroup A $(n=5)$ : crowns were cemented on abutments using resin based temporary cement $\left(\right.$ Dentotemp $\left.^{\mathrm{TM}}\right)$ and Subgroup B $(\mathrm{n}=5)$ : crowns were cemented on abutments using non eugenol zinc oxide temporary cement (Tempbond $\mathrm{NE}^{\mathrm{TM}}$ ).

Dummy implants were inserted in acrylic resin using paralleling device, titanium abutments where screwed on the dummy implants using $1.25 \mathrm{~mm}$ 
diameter Hex and tightened with a precise adjustable torque wrench to a $35 \mathrm{Ncm}$.Titanium abutments were sprayed with a reflective spray and digitally scanned using desktop scanner.

The Crowns were then designed as premolars and adjusted on the designing software to fit on the abutments, then mesial and distal wings were added to aid in the retention test as shown in figure 1.

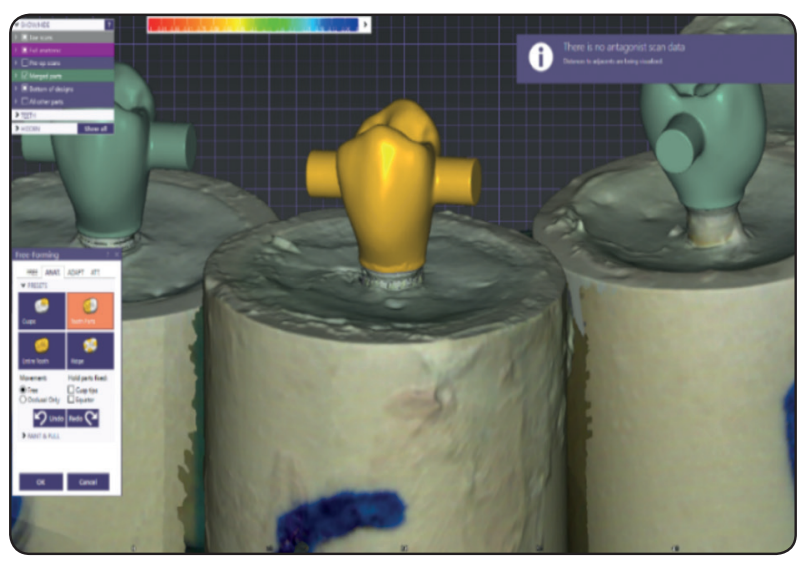

Fig. (1) Computer aided design crowns with two cylindrical extensions

The design was saved as STL file and sent to the milling machine software where the material was selected and the milling started. VITA ENAMIC ${ }^{\text {TM }}$ crowns were separated from the blocks after milling was finished, fitting was checked on the abutments to give its final shape as shown in figure 2(a). BruxZir ${ }^{\mathrm{TM}}$ Solid zirconia crowns were also separated from the blanks then placed under drying lamp for 45 minutes, then sintered for 6 hours in the sintering furnace at $1550^{\circ} \mathrm{C}$. The crown was left in the furnace for cooling to a temperature below $200^{\circ} \mathrm{C}$, giving its final shape, then fitness on the titanium abutments was checked as shown in figure 2(b).

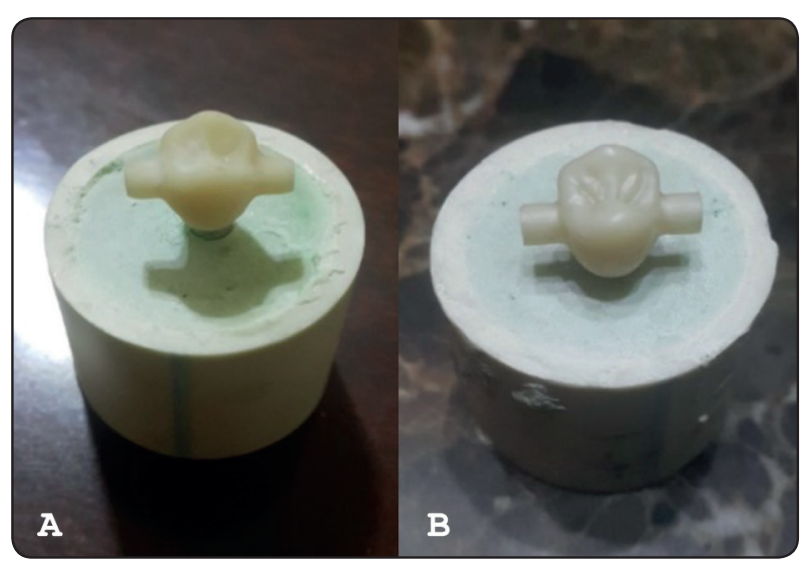

Fig. (2) (a) VITA ENAMIC ${ }^{\text {TM }}$ crown, (b) Zirconia crown

Cements were mixed using the manufacturer instructions and applied on the fitting surfaces of the crowns in each group, Crowns were pressed on the abutments by a loading device under $5 \mathrm{~kg}$ weight ${ }^{5}$, after setting time the samples were ready for testing. Retention was measured by Nexygen ${ }^{\mathrm{TN}}$ Materials Testing Machine ${ }^{6}$ (Model LRX-Plus, Lloyd Instruments, Fareham, UK), Data were recorded using computer software (Nexygen-MT-4.6; Lloyd Instruments) as shown in figure 3.

Numerical data were explored for normality by checking the data distribution, calculating the mean and median values and using KolmogorovSmirnov and Shapiro-Wilk tests. Data showed parametric distribution so; it was represented by mean and standard deviation (SD) values. Two-way ANOVA was used to study the effect of different tested variables and their interaction on retention. Independent t-test was used for different intergroup comparisons. The significance level was set at $\mathrm{P}<$ 0.05 . Statistical analysis was performed with IBM $^{\circledR}$ SPSS $^{\circledR}$ Statistics Version 24 for Windows. 


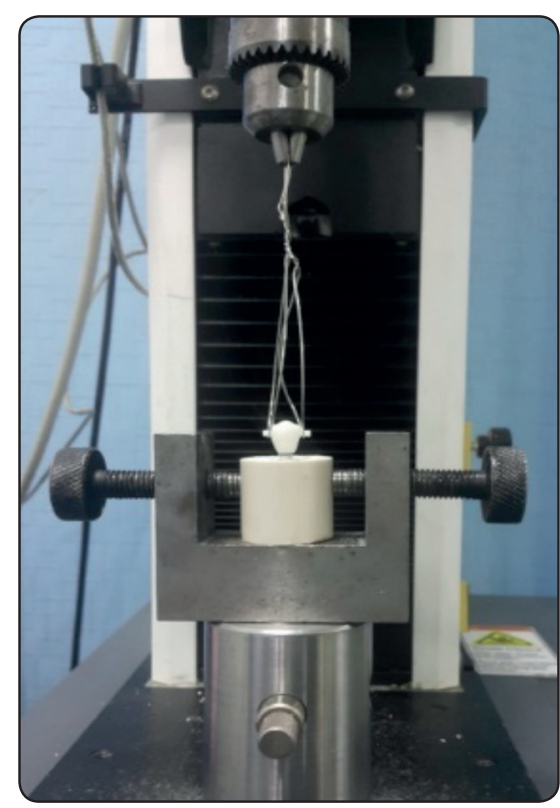

Fig. (3) Retention measurement procedure

\section{RESULTS}

A) Effect of different variables and their interaction: Effect of different variables and their interaction on retention were presented in table (1). Type of temporary cement had a significant effect on retention while type of restoration as well as its interaction with the type of temporary cement had no significant effect.

TABLE (1): Effect of different variables and their interactions on retention

\begin{tabular}{|l|l|}
\hline \multicolumn{1}{|c|}{ Source } & P-value \\
\hline Type of restoration & $0.722 \mathrm{~ns}$ \\
\hline Type of temporary cement & $0.007 *$ \\
\hline $\begin{array}{l}\text { Type of restoration * Type of } \\
\text { temporary cement }\end{array}$ & $0.404 \mathrm{~ns}$ \\
\hline
\end{tabular}

*; Significant $(p<0.05)$ ns; non-significant $(p>0.05)$

B) Effect of Type of restoration: Mean, Standard deviation (SD) for maximum load $(\mathrm{N})$ for different types of the restoration were presented in table (2). Yttrium stabilized tetragonal zirconia (II) had a non-significantly higher $($ mean \pm SD) value than Polymer infiltrated hybrid glass ceramic (I).

TABLE (2): Mean \pm standard deviation (SD) of retention for different types of restorations

\begin{tabular}{|c|c|c|}
\hline \multicolumn{2}{|c|}{ Type of restoration } & \\
\cline { 1 - 2 } $\begin{array}{c}\text { Polymer infiltrated } \\
\text { hybrid glass ceramic } \\
\text { (I) }(\text { mean } \pm \text { SD) }\end{array}$ & $\begin{array}{c}\text { Yttrium stabilized } \\
\text { tetragonal zirconia } \\
\text { (II) }\end{array}$ & \\
\hline $135.82 \pm 29.40$ & $139.26 \pm 21.44$ & $0.768 \mathrm{~ns}$ \\
\hline
\end{tabular}

C) Effect of type of temporary cement: Mean, Standard deviation (SD) for maximum load (N) for different types of temporary cement were presented in table (3). Resin based cement (A) had a significantly higher $($ mean \pm SD) value than Eugenol Free Zinc oxide cement (B).

TABLE (3): Mean \pm standard deviation (SD) of retention for different types of temporary cement

\begin{tabular}{|c|c|c|}
\hline \multicolumn{2}{|c|}{ Type of temporary cement } & \multirow{2}{*}{ P-value } \\
\cline { 1 - 2 } $\begin{array}{c}\text { Resin based cement } \\
\text { (A) }(\text { mean } \pm \text { SD) }\end{array}$ & $\begin{array}{c}\text { Eugenol free Zinc } \\
\text { oxide cement }(B) \\
(\text { mean } \pm \text { SD) }\end{array}$ & \\
\hline $152.27 \pm 15.06$ & $122.81 \pm 24.94$ & $0.005^{*}$ \\
\hline
\end{tabular}

*; Significant $(p<0.05) n s ;$ non-significant $(p>0.05)$

D) Effect of types of temporary cement within each type of restoration: (IA) group showed a non-significantly higher (mean $\pm \mathrm{SD}$ ) value than (IB) while (IIA) showed a significantly higher $($ mean \pm SD) value than (IIB) group.

E) Effect of types of restoration within each type of temporary cement: (IIA) group as well as well as (IB) group showed a non-significantly higher $($ mean \pm SD) value than (IA) and (IIB) groups respectively. 


\section{DISCUSSION}

Cement retained restorations are commonly used as one implant superstructure solution, however reaching a balance between the need for enough retention and easy retrievability is always an issue to be considered, therefore the focus of our study was on retention of cement retained implant supported crowns.

In our study, two types of temporary cements were used to compare their retention values when used with all ceramic implant supported restorations; the non-eugenol zinc oxide type which has been used for long time for temporary cementation of restorations over implants and natural teeth, and the resin based temporary cement which was designed for long term provisional cementation.

Commercially pure titanium abutments where used since commercially pure titanium has been widely used as an abutment material in implant therapy because of its suitable biocompatibility and mechanical properties. ${ }^{7}$

In our study, CAD-CAM technology was used for crowns design and fabrication to allow convenient and standardized manufacturing of all crowns with the same desired measurements.

Zirconia is one of the most commonly used all ceramic materials due to its high strength, fracture toughness, biocompatibility, and excellent esthetics, especially with the introduction of the new translucent CAD-CAM zirconia blanks, making it perfect to use in our study where crowns samples are milled as premolars. Additionally, with the introduction of the new polymer infiltrated hybrid glass ceramics as CAD-CAM blocks for fabricating implant supported restorations, many studies have been done to test its properties in vivo and in vitro because of their excellent esthetics, sufficient strength, high resilience and good shock absorbing capacity. In our study, polymer infiltrated hybrid glass ceramic crowns have been used to compare their retention with zirconia using temporary cements.

All the crowns were pressed on the abutments by a loading device under $5 \mathrm{~kg}$ weigh as done previously by Nejatidanesh et al. ${ }^{8}$ to be sure that all crowns were pressed with the same force until cements reaches their final setting.

In our study, uni-axial tensile force test was selected, to give a better simulation to the clinical environment, similar to a previous study done by Eun-Cheol Shin et al. ${ }^{9}$, where they evaluated retention forces using metalic copings cemented using provisional cements over implant abutments.

Regarding the results of our study, it was found that the resin based temporary cement had significantly higher retentive values than that of the non-eugenol temporary cement which could be explained by the presence of phosphate ester group of acidic monomer in the resin based temporary cement which results in increasing the bonding strength of the cement with the all ceramic crown and the titanium abutment as was previously proven by Barbosa et $\boldsymbol{a l}^{10}{ }^{10}$ in their study of resin cement retention on zirconia supported by titanium abutments.

On the other hand, the type of crown material had non-significant effect on the retention of the restoration, which might be due to the fact that the cements used in our study are temporary and they do not truly adhere to the restoration like permanent cements, also no surface treatment was done to the restoration as was instructed by the cements manufacturers.

In order to achieve adhesion to polymer infiltrated hybrid glass ceramic (VITA ENAMIC ${ }^{\text {TM }}$ ) restorations, they should undergo surface treatment, since they rely mainly on surface treatment in the adhesion process, in our study no surface treatment was made according to the cements manufacturer instructions that stats that no surface treatment is needed when using temporary cements. 
Accordingly, in our study, resin based temporary cement showed a non-significantly higher value than non-eugenol temporary cement when used with polymer infiltrated hybrid glass ceramic crowns which was also proved by Carnaggio, Thomas $V$., et al. ${ }^{5}$ who tested the retention of temporary cements on implant supported restorations without surface treatment and found no significant difference in retentive values between them when used with feldspathic porcelain crowns.

On the contrary, another study by Rohr, Nadja, Brunner, et al. ${ }^{11}$ found that resin based temporary cement showed significantly higher retentive values than those of the zinc oxide non eugenol temporary cement when used with polymer infiltrated hybrid glass ceramic crowns supported by one piece zirconia implants ${ }^{11}$. However, in their study they have done surface treatment to the fitting surfaces of the crowns, which maybe the reason of the significant higher retentive values of the resin based temporary cement than those of the zinc oxide non eugenol temporary cement.

As for the retention of zirconia crowns using resin based temporary cement, our results showed significantly higher values than those when used with zinc oxide non eugenol temporary cement which was previously proved by Kokubo, Y., Kano, T., Tsumita, M., et al. ${ }^{12}$ who tested the retention of zirconia copings on implant abutments cemented with different types of provisional luting agents. In their study they concluded that in case of cementing zirconia restorations over implants using provisional cements, different provisional luting agents resulted in different retentive forces with or without surface treatment.

One explanation is that zirconia is a polycrystalline ceramic, where surface treatment may be less crucial in adhesion process. In our study, no surface treatment was done, making the retention rely mainly on the properties of each cement, thus the resin based temporary cement show a significantly higher retention value than non-eugenol temporary cement when used with zirconia crowns due to the presence of phosphate ester group of acidic monomer in the resin based temporary cement.

Conversely, another study done by Nejatidanesh, Farahnaz Savabi, et al. ${ }^{8}$ disagreed with our study results, where they found no significant difference in retentive value between resin based temporary cement and zinc oxide non eugenol temporary cement when used with zirconia copings supported by titanium implant abutments. Although no surface treatment was done to the crowns similar to our study, yet the difference between their study and ours might be due that their copings were incubated and conditioned in artificial saliva and thermal cycled before retention measurement, which might be a limitation in our study where aging conditions simulating intraoral environment were absent.

One limitation of this study was the use of a constant dislodgment force. The retention of implant supported restorations should be tested by a high impact force in a short duration of time ${ }^{13}$. On the other hand, intraoral occlusal forces are dynamic in nature not a static monotonic load. Cement retention may be different under fatigue forces compared to static load application. In vitro studies such as our study do not produce the same results as clinical studies, and their outcomes should be interpreted with caution.

It is worth mentioning that studies evaluating cements retention are generally carried out with thermo-cycling or water storage to simulate oral conditions. While some studies have shown that aging processes destroy the effects of cements bonding due to hydrolytic degradation ${ }^{14}$, some have stated that there are no significant differences in bond strength before and after the aging process ${ }^{15}$. The current study was carried out in dry conditions, as the aim of this study was to evaluate early failure between the selected temporary cements when used with all ceramic implant supported restorations. 
More research should be done to prove whether or not surface treatment of fitting surface especially of polymer infiltrated hybrid glass ceramic restorations and aging conditions can affect their retention when supported by titanium implant abutments using temporary cements.

\section{CONCLUSIONS}

Within the limitations of this study:

1. Resin based temporary cement showed a higher retentive values with each crown material.

2. There was no much difference in retentive values between Polymer infiltrated hybrid glass ceramic crowns and zirconia crowns in case of using the same temporary cement.

3. The retentive values of the resin based temporary cement was significantly higher than those of the zinc oxide non eugenol temporary cement when used with zirconia crowns, while retentive values of the resin based temporary cement was non significantly higher than those of the zinc oxide non eugenol temporary cement when used with Polymer infiltrated hybrid glass ceramic crowns.

\section{REFERENCES}

1. Sailer I, Mühlemann S, Zwahlen M, Hämmerle CHF, Schneider D. Cemented and screw-retained implant reconstructions: A systematic review of the survival and complication rates. Clin Oral Implants Res. 2012; 23 (SUPPL.6):163-201. doi:10.1111/j.1600-0501.2012.02538.x.

2. Dudley JE, Richards LC, Abbottf JR. Retention of cast crown copings cemented to implant abutments. Aust Dent J. 2008;53(4):332-339. doi:10.1111/j.18347819.2008.00075.x.

3. Alvarez-Arenal A, Gonzalez-Gonzalez I, DeLlanosLanchares H, Brizuela-Velasco A, Ellacuria-Echebarria $\mathrm{J}$. The selection criteria of temporary or permanent luting agents in implant-supported prostheses: in vitro study. J Adv Prosthodont. 2016;8(2):144-149. doi:10.4047/ jap.2016.8.2.144.
4. Nagasawa Y, Hibino Y, Nakajima H. Retention of crowns cemented on implant abutments with temporary cements. Dent Mater J. 2014;33(6):835-844. doi:10.4012/dmj. 2014-100.

5. Carnaggio T V., Conrad R, Engelmeier RL, et al. Retention of CAD/CAM All-Ceramic Crowns on Prefabricated Implant Abutments: An In Vitro Comparative Study of Luting Agents and Abutment Surface Area. J Prosthodont. 2012;21(7):523-528. doi:10.1111/j.1532849X.2012.00847.x.

6. Johnson GH, Lepe X, Patterson A, Schäfer O. Simplified cementation of lithium disilicate crowns: Retention with various adhesive resin cement combinations. J Prosthet Dent. 2017:1-7. doi:10.1016/j.prosdent.2017.07.012.

7. Menon NS, Kumar GPS, Jnanadev KR, Satish Babu CL, Shetty S. Assessment and comparison of retention of zirconia copings luted with different cements onto zirconia and titanium abutments: An in vitro study. J Indian Prosthodont Soc. 2016;16(2):136-141. doi:10.4103/09724052.176540 .

8. Nejatidanesh F, Savabi O, Shahtoosi M. Retention of implant-supported zirconium oxide ceramic restorations using different luting agents. Clin Oral Implants Res. 2013; 24(A100):20-24. doi:10.1111/j.1600-0501.2011.02358.x.

9. Keum E-C, Shin S-Y. A comparison of retentive strength of implant cement depending on various methods of removing provisional cement from implant abutment. J Adv Prosthodont. 2013;5(3):234-240. doi:10.4047/ jap.2013.5.3.234.

10. CAMARGO FP, ANDREATTA FILHO OD, BOTTINO MA. Durability of bond strength between titanium alloy and resin cement Durabilidade da resistência de uni\ ao entre uma liga de titânio e um cimento resinoso S'rlvia Helena BARBOSA. Cienc Odontol Bras. 2008;11(1):1318. http://www.fosjc.unesp.br/cob/artigos/v11n1_02.pdf.

11. Rohr N, Brunner S, Märtin S, Fischer J. Influence of cement type and ceramic primer on retention of polymer-infiltrated ceramic crowns to a one-piece zirconia implant. J Prosthet Dent. 2017:1-8. doi:10.1016/j.prosdent.2017.02.002.

12. Kokubo Y, Kano T, Tsumita M, Sakurai S, Itayama A, Fukushima S. Retention of zirconia copings on zirconia implant abutments cemented with provisional luting agents. J Oral Rehabil. 2010;37(1):48-53. doi:10.1111/ j.1365-2842.2009.02013.x. 
13. Mehl C, Harder S, Wolfart M, Kern M, Wolfart S Retrievability of implant-retained crowns following cementation. Clin Oral Implants Res. 2008;19(12):13041311. doi:10.1111/j.1600-0501.2008.01587.x.

14. Li R. Development of a ceramic primer with higher bond durability for resin cement. J Oral Rehabil. 2010;37(7):560568. doi:10.1111/j.1365-2842.2010.02060.x.
15. Amaral R, Özcan M, Valandro LF, Balducci I, Bottino MA. Effect of conditioning methods on the microtensile bond strength of phosphate monomer-based cement on zirconia ceramic in dry and aged conditions. J Biomed Mater Res- Part B Appl Biomater. 2008;85(1):1-9. doi:10.1002/ jbm.b.30908. 